\title{
AVALIAÇÃO DE RISCO PARA O DESENVOLVIMENTO DE ÚLCERAS DE PRESSÃO EM PACIENTES INTERNADOS EM CENTRO DE TERAPIA INTENSIVA
}

[Risk Assessment to pressure ulcer development scales in patients hospitalized in intensive care unit]

Luciana Magnani Fernandes* Elizabeth Braz**

RESUMO: Buscar maneiras de avaliar o risco de desenvolvimento de úlceras como consolidação de uma medida preventiva foi um objetivo que levou alguns pesquisadores a criarem instrumentos que possibilitassem a predição do risco. Este trabalho tem como objetivo, comparar o poder preditivo para o desenvolvimento de úlceras de pressão das Escalas de Braden e Waterlow em pacientes internados em Centro de Terapia Intensiva. Para tal foi realizado um estudo descritivo comparativo prospectivo, com uma abordagem quantitativa, que teve uma amostra de 23 pacientes internados na unidade. Com relação ao valor preditivo das escalas, a escala de Braden apresentou melhor performance, se comparada com a escala de Waterlow.

PALAVRAS-CHAVE: Úlcera de pressão; Prevenção e Controle; Pacientes hospitalizados.

\section{INTRODUÇÃO}

As úlceras de pressão são definidas como "áreas localizadas de necrose tissular que tendem a se desenvolver quando o tecido mole é comprimido entre a proeminência óssea e uma superfície externa por um longo período de tempo" (BRYANT, 1992: 110).

Os determinantes críticos na formação da úlcera de pressão foram definidos por BRADEN; BERGSTROM (1987), como sendo a intensidade e duração da pressão sobre regiões do corpo e a capacidade da pele e tecidos subjacentes para tolerá-los.

Índices de pressão exagerados, em determinada área do corpo, causam oclusão capilar, que desencadeia diminuição do suprimento sangüíneo, de nutrientes e de oxigênio aos tecidos. A quantidade de pressão externa necessária para causar o colapso capilar deve exceder a pressão do capilar que, segundo GUYTON (1981), é de

* Mestre em Enfermagem, Docente do Colegiado do Curso de Enfermagem do UNIOESTE - Universidade Estadual do Oeste do Paraná.

** Mestre em Enfermagem, Docente do Colegiado do Curso da UNIOESTE Universidade Estadual do Oeste do Paraná.
$35 \mathrm{mmHg}$. A duração da pressão é fator fundamental na formação de úlceras, pois quanto maior o tempo de exposição à pressão, maiores serão os danos aos tecidos.

A tolerância tissular está relacionada à capacidade dos tecidos de suportar a pressão exercida nestes. Tolerância tissular é, portanto, um fator que descreve a condição ou integridade da pele e estruturas de suporte que possam influenciar na capacidade da pele de redistribuir e aplicar a pressão (BRYANT, 1992).

Podemos citar como fatores de risco para o desenvolvimento da úlcera de pressão: diminuição da percepção sensorial; diminuição da mobilidade; umidade; fricção; cisalhamento; deficiência nutricional; idade avançada; níveis pressóricos baixos; dimunuição do nível de consciência; condições sistêmicas crônicas; fatores relacionados à higiene da pele entre outros (FERNANDES, 2000).

De acordo com NORRIS (1982), outro fator relevante no desenvolvimento de úlceras de pressão é o diagnóstico médico, como exemplo: câncer, diabetes mellitus, infecções, cardiopatias.

Os locais mais suscetíveis ao desenvolvimento de úlceras de pressão são as proeminências ósseas devido à distribuição desigual de peso e excesso de pressão nessas áreas. Úlceras de pressão podem ocorrer sobre o sacro, ísqueo, trocanter do fêmur, cotovelo, calcâneo, escápula, osso occipital, esterno, costelas, crista ilíaca, patela, maléolos, porém devido à maior concentração de proeminências ósseas e da distribuição desigual de peso, a maior parte dessas úlceras ocorrem na parte inferior do corpo (MAKLEBUST; SIEGGREEN, 1996).

Buscar maneiras de avaliar o risco de desenvolvimento de úlceras como consolidação de uma medida preventiva foi um objetivo que levou alguns pesquisadores a criarem instrumentos que possibilitassem a predição do risco.

Duas escalas de avaliação de risco bastante conhecidas e utilizadas são a de Braden e da Waterlow (MAKLEBUST; SIEGGREEN, 1996), onde a pontuação final deverá representar o grau de risco do doente, fornecendo dados mais objetivos para a observação e avaliação que o enfermeiro poderá realizar no processo de prevenção. 
Segundo MAKLEBUST; SIEGGREEN (1996), testes de confiabilidade e validade apontam a Escala de Braden e a de Waterlow como apropriadas para avaliação do risco para o desenvolvimento de úlcera de pressão e servem como instrumentos proveitosos no estabelecimento precoce de prevenção e na elaboração do cuidado em populações de risco.

A Escala de Braden é considerada a mais completa e tem sido amplamente utilizada por pesquisadores. Fornece seis parâmetros para avaliação também chamados de subescalas: 1- percepção sensorial; 2- umidade; 3- atividade; 4mobilidade; 5- nutrição; 6- fricção e cisalhamento. Cada subescala tem uma pontuação que varia entre 1 a 4 . A somatória total fica entre os valores de 6 a 23. Quanto maior a pontuação final, menor será o risco de desenvolvimento de úlceras de pressão. Segundo MAKLEBUST; SIEGGREEN (1996), a somatória total menor ou igual a 16 denota risco para o desenvolvimento de úlcera de pressão.

A Escala de Waterlow consiste de 10 itens: estrutura/ peso e altura; continência; tipo de pele em áreas de risco; mobilidade; sexo e idade; apetite; má nutrição tecidual; déficit neurológico; cirurgias / traumas; medicação. Cada item tem uma pontuação própria, com valores que variam entre 0 a 5 . $\mathrm{Na}$ avaliação do paciente deve-se considerar, segundo a autora, o escore acima de 10 como preditor de risco para o desenvolvimento de úlceras de pressão (WATERLOW, 1992).

A avaliação do risco através da utilização desses instrumentos deve ser realizada na admissão e periodicamente durante a hospitalização. Essa periodicidade deve obedecer à gravidade do quadro do paciente. Para pacientes internados em unidades de terapia intensiva, recomenda-se o uso diário, devido à instabilidade do paciente.

As escalas são instrumentos que auxiliam os profissionais no julgamento clínico e não substitui o mesmo. Os fatores de risco não mensurados pela escala precisam ser integrados na avaliação.

Este estudo teve como objetivo comparar o poder preditivo para o desenvolvimento de úlceras de pressão das Escalas de Braden e Waterlow em pacientes internados em Centro de Terapia Intensiva.

\section{METODOLOGIA}

Trata-se de um estudo descritivo comparativo. $O$ estudo descritivo comparativo examina e descreve diferenças nas variáveis em 2 ou mais grupos que ocorrem naturalmente.
A população foi composta de pacientes internados no Centro de Terapia Intensiva de um hospital público de uma cidade do interior do Estado do Paraná. A amostra compreendeu 23 (vinte e três) pacientes que se encaixaram nos critérios de inclusão estabelecidos para este estudo. Os critérios de inclusão foram: pacientes internados no Centro de Terapia Intensiva do referido hospital; pacientes com previsão de permanência de 2 dias ou mais; pacientes com idade igual ou superior a 18 anos e pacientes com a pele íntegra, ou seja, sem a presença de úlceras de pressão.

Os dados foram coletados pelos pesquisadores, através de instrumento desenvolvido especificamente para este fim. Inicialmente, foi realizada a primeira avaliação do paciente no dia da internação (até 24 horas após a admissão). Esta avaliação constou de aplicação da escala de Braden e da escala de Waterlow para a determinação do grau de risco para o desenvolvimento de úlcera de pressão que o paciente apresentava, assim como inspeção das condições da pele, estado de saúde e medidas preventivas de úlcera de pressão realizadas.

A cada 48 horas o paciente foi reavaliado até que o mesmo recebeu alta do Centro de Terapia Intensiva ou desenvolveu úlceras de pressão. A ocorrência de úlcera de pressão foi discutida com a equipe de saúde da unidade de internação hospitalar e registrado como dados da pesquisa.

As variáveis estudadas foram: idade; cor; sexo; diagnóstico médico; presença de úlcera de pressão; localização da úlcera; estágio da úlcera; escores das escalas de Braden e Waterlow; pressão arterial; hematócrito; hemoglobina; temperatura corporal; uso de drogas; tipo de dieta; ocorrência cirúrgica com mais de duas horas de duração; presença de sondagem vesical, nasogástrica, ventilação mecânica, drenagem torácica, diálise peritonial e fixador externo em membro inferior.

Foram obtidos consentimentos, do paciente ou familiares, para a obtenção de dados. O trabalho teve aprovação do Comitê de Ética da UNIOESTE - Universidade Estadual do Oeste do Paraná.

Após a coleta de dados, foram obtidos os valores de escores das escalas para a determinação do risco dos pacientes e realizada comparação dos instrumentos de avaliação de risco especificamente para pacientes internados em Centro de Terapia Intensiva.

Para maior confiabilidade da análise dos dados, os mesmos foram submetidos a testes de sensibilidade e especificidade. 


\section{APRESENTAÇÃO E DISCUSSÃO DOS DADOS}

Um total de 23 sujeitos foram estudados. A idade desses variou entre 18 e 84 anos (média $=48.4$ anos), eram predominantemente brancos (91.3\%) e homens (60.9\%). Os diagnósticos mais freqüentes foram Acidente vascular cerebral (21.7\%) e politraumatismos (17.4\%).

Foram instituídas terapias de enfermagem preventivas para úlcera de pressão para todos os pacientes que fizeram parte da amostra, sendo elas a mudança de decúbito de duas em duas horas e a lubrificação da pele com óleo de girassol. Essas medidas são rotineiras na referida Unidade de Internação e não houve avaliação da aplicação dessas medidas nos resultados obtidos.

Dos referidos sujeitos, 12 (52.2\%) desenvolveram 21 úlceras de pressão (média = 1.75 úlcera por sujeito). O local mais atingido pelas úlceras de pressão foi a região sacra, sendo 8 em estágio I e 2 em estágio II, seguido do calcâneo com 7 úlceras em estágio I e 1 em estágio II. Em maléolo foram observadas 2 úlceras, uma em estágio I e uma em estágio II e em trocanter apenas 1 úlcera foi encontrada e em estágio II.

A idade dos pacientes que desenvolveram úlceras de pressão esteve entre 18 e 84 anos (média = 42.7 anos), sendo o sexo masculino o predominante (30.4\%) e da cor branca (43.5\%). Com relação aos pacientes que não desenvolveram úlceras, a idade destes esteve entre 18 e 77 anos (média = 49.4 anos), sendo também o sexo masculino (30.4\%) e de cor branca (47.8\%) os predominantes (tabela 1).

Analisando os escores das escalas, observamos (tabela 1) que, entre os pacientes que desenvolveram úlceras, os escores da escala de Braden estiveram entre 9 e 13 (média = 11.0) e de Waterlow entre 13 e 25 (média = 16.6). Entre os pacientes que não desenvolveram úlceras, os escores da escala de Braden variaram entre 9 e 17 (média = 10.6) e da escala de Waterlow entre 8 e 21 (média = 16.20).

Não houve diferença significativa na idade, sexo, cor e escores das escalas entre aqueles que desenvolveram úlceras de pressão e aqueles que não desenvolveram.

Com relação às patologias de base que os pacientes estudados apresentaram, observamos que o acidente vascular cerebral (25\%), politraumatismos (16.7\%) e oclusão arterial em membros inferiores (16.7\%) foram mais freqüentes nos pacientes que desenvolveram úlceras. Nos pacientes que não desenvolveram úlceras, as patologias mais freqüentes foram o acidente vascular cerebral (18.2\%), politraumatismos $(18.2 \%)$ e traumatismo craniano (18.2\%).
TABELA 1 - CARACTERÍSTICAS DOS SUJEITOS QUE DESENVOLVERAM ÚLCERAS DE PRESSÃO (PS+) E DOS QUE NÃO DESENVOLVERAM (PS-). CASCAVEL, 2001

\begin{tabular}{|c|c|c|}
\hline CARACTERISTICAS & PS (+) & PS (-) \\
\hline & $N=12$ & $N=11$ \\
\hline \multicolumn{3}{|l|}{ Idade (anos) } \\
\hline$X \pm D P$ & $42.17 \pm 15.82$ & $49.4 \pm 22.54$ \\
\hline Extensão & $(18-84)$ & $(18-77)$ \\
\hline \multicolumn{3}{|l|}{ Escore Braden } \\
\hline$X \pm D P$ & $11.0 \pm 1.96$ & $10.6 \pm 2.61$ \\
\hline Extensão & $(9-13)$ & $(9-17)$ \\
\hline \multicolumn{3}{|l|}{ Escore Waterlow } \\
\hline$X \pm D P$ & $16.6 \pm 3.02$ & $16.20 \pm 4.95$ \\
\hline Extensão & $(13-25)$ & $(8-21)$ \\
\hline Sexo & Número \% & Número $\%$ \\
\hline Masculino & 730.4 & 730.4 \\
\hline Feminino & 521.7 & 417.4 \\
\hline Cor & Número \% & Número $\%$ \\
\hline Branca & 1043.5 & 1147.8 \\
\hline Negra & 28.7 & -- -- \\
\hline
\end{tabular}

Dentre os pacientes que desenvolveram úlceras de pressão (Tabela 2), a pressão sistólica variou entre 50 a 160 $\mathrm{mmHg}$ (média 96.6), a pressão diastólica variou entre 20 a $100 \mathrm{mmHg}$ (média 61.6), o hematócrito variou entre 22.8 a 45.6 (média 34.8), a hemoglobina entre 7.40 a 15.20 (média 11.28) e a temperatura corporal entre 36.2 a 39.5 graus centígrados (média 38.4).

Observamos que dentre os pacientes que não desenvolveram úlceras, a pressão sistólica variou entre 80 a 220 mmHg (média 116.0), a pressão diastólica entre 40 a 110 $\mathrm{mmHg}$ (média 74.0), o hematócrito entre 8.70 a 47.0 (média 35.9), a hemoglobina entre 3.0 a 15.9 (média 11.9) e a temperatura corporal entre 36.6 a 39.5 graus centígrados (média 37.9).

Pudemos comprovar que os fatores que apresentaram significância foram os valores de pressão sistólica e diastólica e temperatura corporal.

O uso de algumas drogas é sugerido por alguns autores como fatores de risco para o desenvolvimento de úlceras de pressão, devido aos seus efeitos sobre o organismo. na população que desenvolveu úlceras o uso mais expressivo de medicação foi o do corticóide em $66.7 \%$ desta e de hipotensores em 33.3\%. Fenobarbital, analgésicos e sedativos foram utilizados por $25 \%$ (cada medicação) destes pacientes e manitol, antiinflamatórios e dopamina foram utilizados em 8.3\% (cada medicação). 
Na população que não desenvolveu úlceras, o uso mais expressivo de medicações foi de corticóide em $63.7 \%$ desta e de sedativos em $36.4 \%$. O uso de hipotensores, analgésicos, manitol e antiinflamatório atingiram um total de $27.3 \%$ (cada medicação) de pacientes e o fenobarbital e dopamina um total de $9.1 \%$ (cada medicação) destes pacientes.

$\mathrm{O}$ aspecto nutricional é outro item relevante para a manutenção da integridade cutânea e para a prevenção de úlceras de pressão. Foi realizada uma análise da dietoterapia que os pacientes estavam recebendo e observamos que a maioria dos pacientes mantiveram-se em jejum durante 0 estudo. Nos pacientes que desenvolveram úlceras, $83 \%$ estavam em jejum, $8.3 \%$ recebia dieta pastosa e $8.3 \%$ dieta leve. Nos pacientes que não desenvolveram úlceras, $72.7 \%$ mantiveram-se em jejum, $18.2 \%$ recebiam dieta líquida (por via oral) e $9.1 \%$ dieta leve. Nenhum dos pacientes estudados receberam dieta por sonda ou nutrição parenteral.

TABELA2 - COMPARAÇÃO DE VARIÁVEIS EM PACIENTES QUE DESENVOLVERAM ÚLCERAS DE PRESSÃO (PS+) E NOS QUE NÃO DESENVOLVERAM (PS-). CASCAVEL, 2001.
VARIÁVEIS

PS (+)

PS (-)

\section{Pressão Sistólica}

\section{$\mathrm{X} \pm \mathrm{DP}$}

Extensão

$96.6 \pm 3.24$

$(50-160)$

$116.0 \pm 1.74$

(80-220)

\section{Pressão diastólica}

$\mathrm{X} \pm \mathrm{DP}$

Extensão

Hematócrito

$\mathrm{X} \pm \mathrm{DP}$

Extensão

$34.8 \pm 6.69$

$(22.8-45.6)$

$35.9 \pm 5.50$

(8.70-47.0)

\section{Hemoglobina}

$\mathrm{X} \pm \mathrm{DP}$

Extensão

Temperatura

$\mathrm{X} \pm \mathrm{DP}$

Extensão

$38.4 \pm 0.6$

(36.2-39.5)
$74.0 \pm 1.49$

$(40-110)$
Segundo STOTTS (1999), procedimentos cirúrgicos com duração de duas horas ou mais são considerados fatores de risco para o desenvolvimento de úlceras de pressão devido ao período prolongado de pressão exercida nos tecidos ocasionado pela ausência de mudança no decúbito destes além da superfície onde está deitado ser inadequada.

Dos pacientes que desenvolveram úlceras, 50\% (6 pacientes) foram submetidos a procedimentos cirúrgicos prolongados. Craniotomia foi realizado em 50\% destes, embolectomia em 33.3\% e exploração arterial em 16.7\%. Dos pacientes que não desenvolveram, apenas $18.2 \%$ foram submetidos a estes tipos de procedimentos. Gastrectomia em 50\% destes e osteossíntese de fêmur em 50\%.

Outros procedimentos significantes foram relacionados para maior compreensão do fenômeno que envolveu a formação de úlceras de pressão. Entre os pacientes que desenvolveram úlceras, 91,7\% foram submetidos à sondagem vesical, $66.7 \%$ a sondagem nasogástrica, $50 \%$ a ventilação mecânica, $16.7 \%$ a drenagem torácica, 8.3\% a diálise peritonial e $8.3 \%$ ao fixador externo em membro inferior.

Entre os que não desenvolveram úlceras, 100\% foram submetidos a sondagem vesical, $81.2 \%$ a sondagem nasogástrica e $36.4 \%$ a ventilação mecânica.

O Quadro 1 e 2 mostram os resultados encontrados através da aplicação dos testes de validade preditiva da escala de Braden e da escala de Waterlow, ou seja, sensibilidade, especificidade, valor preditivo + e valor preditivo -. Os referidos testes foram aplicados em cada um dos escores encontrados nas avaliações realizadas nos pacientes que fizeram parte deste estudo. Para este fim foram utilizadas os testes indicados por BERGSTROM, DEMUTH e BRADEN (1987).

Especificamente o Quadro 1 mostra a distribuição dos testes de validade preditiva da escala de Braden. Os dados referem-se a sete escores que compuseram as avaliações dos pacientes, visto que alguns escores se repetem em vários pacientes.

Os escores que mais se destacaram foram o 15 e o 13, revelando uma melhor performance nos testes de validade preditiva, destacando o escore 15 como o escore que apresentou maior validade preditiva na população que fez parte deste estudo, com sensibilidade de $100 \%$, especificidade de $100 \%$, valor preditivo positivo de $100 \%$ e valor preditivo negativo nulo.

QUADRO 1 - DISTRIBUIÇÃO DOS TESTES DE VALIDADE PREDITIVA REALIZADOS NOS ESCORES DA ESCALA DE BRADEN. CASCAVEL, 2001

\begin{tabular}{|c|c|c|c|c|}
\hline ESCORE & $\begin{array}{c}\text { SENSIBILIDADE } \\
(\%)\end{array}$ & $\begin{array}{c}\text { ESPECIFICIDADE } \\
(\%)\end{array}$ & $\begin{array}{c}\text { VALOR } \\
\text { PREDITIVO + } \\
(\%)\end{array}$ & $\begin{array}{c}\text { VALOR } \\
\text { PREDITIVO - } \\
(\%)\end{array}$ \\
\hline 9 & 50 & 47 & 33 & 64 \\
10 & 64 & 58 & 58 & 64 \\
11 & 53 & 50 & 67 & 36 \\
12 & 61 & 80 & 91 & 36 \\
13 & 100 & 9 & 55 & 100 \\
15 & 100 & 100 & 100 & 0.9 \\
17 & 52 & 0 & 100 & 0 \\
\hline
\end{tabular}


Ao analisarmos os escores da Escala de Waterlow (Quadro 2), observamos a distribuição dos testes de validade preditiva da escala de Waterlow. Os dados referem-se a sete escores que compuseram as avaliações dos pacientes, visto que alguns escores se repetem em vários pacientes.

Os escores que mais se destacaram foram o 19 e o 20 revelando uma melhor performance nos testes de validade preditiva, destacando o escore 20 como o que apresentou maior validade preditiva na população que fez parte deste estudo, com sensibilidade de $52 \%$, especificidade de $50 \%$, valor preditivo positivo de $92 \%$ e valor preditivo negativo $9 \%$.

QUADRO 2 - DISTRIBUIÇÃO DOS TESTES DE VALIDADE PREDITIVA REALIZADOS NOS ESCORES DA ESCALA DE WATERLOW. CASCAVEL, 2001

\begin{tabular}{|c|c|c|c|c|}
\hline ESCORE & $\begin{array}{c}\text { SENSIBILIDADE } \\
(\%)\end{array}$ & $\begin{array}{c}\text { ESPECIFICIDADE } \\
(\%)\end{array}$ & $\begin{array}{c}\text { VALOR } \\
\text { PREDITIVO + } \\
(\%)\end{array}$ & $\begin{array}{c}\text { VALOR } \\
\text { PREDITIVO - } \\
(\%)\end{array}$ \\
\hline 8 & 0 & 45 & 0 & 91 \\
10 & 0 & 43 & 0 & 82 \\
12 & 0 & 40 & 0 & 73 \\
13 & 29 & 38 & 17 & 56 \\
14 & 38 & 40 & 25 & 55 \\
16 & 33 & 36 & 25 & 45 \\
17 & 45 & 42 & 42 & 45 \\
19 & 46 & 40 & 50 & 36 \\
20 & 52 & 50 & 92 & 9 \\
21 & 50 & 0 & 92 & 0 \\
25 & 52 & 0 & 100 & 0 \\
\hline
\end{tabular}

\section{CONSIDERAÇÕES FINAIS}

A predominância neste estudo foi a faixa etária entre 18-30 anos (26.1\%), pacientes do sexo masculino (60.1\%) e de cor branca (91.3\%). A patologia base mais freqüente foi o Acidente Vascular Cerebral (21.75\%).

Os fatores de risco mais comumente encontrados nos pacientes foram: uso de corticóides (60.83\%); hipotensão arterial (30.4\%); anemia (69.6\%); jejum (78.26\%); procedimento cirúrgico com duração igual ou maior que 2 horas (34.78\%) e hipertermia (21.74\%).

A incidência de úlcera de pressão foi de $52.2 \%$. A localização mais comum destas foi a região sacra (50\%) e a avaliação da gravidade das mesmas através do estadiamento mais comum foi o grau I (77.3\%).

Todos os pacientes receberam como medidas preventivas o uso tópico do óleo de girassol na pele íntegra e mudança de decúbito de 2 em 2 horas.

Entre os pacientes que desenvolveram úlceras de pressão, as faixas etárias dominantes foram 18-30 anos, 51-
60 anos e $61-70$ anos, sendo que $58.3 \%$ destes eram do sexo masculino e $83.3 \%$ de cor branca. As patologias de base mais freqüentes foram Acidente Vascular Cerebral, Politraumatismos e Trombose Arterial em membros inferiores. Esses pacientes tiveram como fatores de risco: hipotensão arterial (33.3\%); anemia (75\%); jejum (83.3\%); procedimento cirúrgico de duas horas ou mais (50\%); hipertermia (25\%) e uso de glucocorticóide (75\%).

A definição dos fatores de risco para úlcera de pressão é um tema que tem sido bastante estudado por pesquisadores e tem demonstrado ser de extrema importância, para a compreensão do processo de desenvolvimento das úlceras e da definição dos indivíduos mais propensos para tal, na tentativa de adoção de medidas precoces com o objetivo de promover a prevenção e controle do problema.

A avaliação do risco permite então a identificação do nível de risco e também os fatores contribuintes para o risco elevado. Esta avaliação pode ser feita através de instrumentos de avaliação, denominados de escalas.

A escala de Braden, segundo os estudos, apresenta maior confiabilidade (sensibilidade e especificidade) na predição de risco para o desenvolvimento de úlceras de pressão.

Em nosso estudo, as escalas de Braden e Waterlow tiveram o mesmo valor preditivo, podendo ambas ser recomendadas para uso em Centro de Terapia Intensiva, numa população semelhante, para a determinação do grau de risco do paciente para o desenvolvimento de úlcera de pressão, o que contribuiria em muito na adoção de medidas preventivas e na identificação dos fatores individuais de risco.

Reconhecemos que novos estudos, com uma amostra maior para uma análise mais ampla de dados, seria recomendada.

ABSTRACT: To look for ways to evaluate the risk for pressure ulcer development like prevention was a researcher purpose whose created some instruments to predict the risk. The purpose this work was to compare the scales Braden and Waterlow pressure ulcer development power predictive. It was used a descriptive, comparative prospective study. The sample was 23 critical pacients hospitalizaded in Intensive Care Unit. The Braden scale showed the better results, if comparaded with Waterlow scale.

KEY WORDS: Pressure ulcer; Prevention and control; impatients. 


\section{REFERÊNCIAS}

1 BERGSTROM, N.; DEMUTH, P.J.; BRADEN B. J. A clinical trial of the Braden Scale for predicting pressure sore risk. Nurs. Clin. North Am., Philadelphia, v. 22, n.2, june 1987.

2 BRADEN, B.; BERGSTROM, N. A conceptual scheme to study of pressure ulcer. Rehabil. Nurs. v. 12, n. 1, Jan/Feb. 1987.

3 BRYANT, R. A. Acute and chronics wounds nursing management. St. Louis: Mosby Year Book, 1992.

4 GUYTON, A C. Fisiologia humana. 5. ed. Rio de Janeiro: Interamericana, 1981

5 FERNANDES, L. M. Úlcera de pressão em pacientes críticos hospitalizados. Uma revisão integrativa da literatura. Ribeirão Preto, 2000. 168 p. Dissertação (Mestrado em Enfermagem Fundamental) - Escola de Enfermagem de Ribeirão Preto da Universidade de São Paulo.
6 MAKLEBUST, J.; SIEGGREEN, M. Pressure ulcer: guidelines for prevention and nursing management. Philadelphia: Springhouse Corporation, $1996304 \mathrm{p}$

7 NORRIS, C. M. Concept clarification in nursing. London: Aspen, 1982.

8 STOTTS, N. A. Risk of pressure ulcer development in surgical patients: a review of the literature. Adv. Wound Care, v. 12, n. 3, p.127-36, 1999.

9 WATERLOW, J. A policy that protects: the Waterlow pressure sore prevention/treatment policy. In: HORNE, E. M.; COWAN, T. Staff nurse's survival guide. 2. ed. London: Mosby, 1992.

Endereço das autoras: Rua Presidente Bernardes 3148 - Centro 85810-130 - Cascavel - PR e-mail: lumagna@terra.com.br 